\title{
Anti-NMDA Encephalitis Cases Secondary to Teratoma and Pregnancy
}

\author{
Robert Perna* \\ Clinical Neuropsychologist, TIRR Memorial Hermann, USA
}

*Corresponding author: Robert Perna, Clinical Neuropsychologist, TIRR

Memorial Hermann, Texas, Medical Center Houston, 2450 Holcombe Blvd \#1,

Houston, TX 77021, USA.

Received Date: September 24, 2018

Published Date: October 12, 2018

\begin{abstract}
Anti-NMDA receptor (anti-NMDAR) encephalitis frequently develops in young women [1] and can be related to an autoimmune reaction to pregnancy or an ovarian teratoma. Severe new onset psychiatric symptoms and/or seizures in young adult women should warrant a potential NMDAR encephalitis differential diagnosis. This manuscript describes two cases of NMDAR encephalitis, one in an individual in the 8th week of gestation and the other case involves encephalitis secondary to ovarian teratoma. Both individuals required hospitalization, had residual short-term memory, and executive system dysfunction, and required post-acute brain injury rehabilitation.
\end{abstract}

\section{Introduction}

Anti-NMDA receptor (anti-NMDAR) encephalitis frequently develops in young women [1] and there is a growing body of research suggesting that anti-NMDAR encephalitis can be triggered by pregnancy or a tumor [2]. Anti-NMDAR encephalitis associated with pregnancy has been increasingly reported [2,3]. Given this phenomenon, this disorder should be considered among the differential diagnoses in this population when there are new onset major psychiatric symptoms. Though this disorder can dramatically affect an individual's behavior, and frequently involves the need for acute hospitalization and various medications, history of anti-NMDAR encephalitis has not prohibited many individuals from achieving their reproductive goals [4]. Despite subsequently delivering a healthy newborn, anti-NMDA encephalitis drastically complicates the situation. There may be a clinical need for various psychotropic medications and anti-convulsant medications and a complex weighing of teratogenic risks.

Anti-N-methyl-D-aspartate receptor (NMDAR) encephalitis is a new category of treatment-responsive encephalitis associated with "anti-NMDAR antibodies," which bind to the NMDA receptors. Overall, almost $50 \%$ of young adult women with this disorder harbor an ovarian teratoma, which presumably has triggered an antibody response to its neural tissue. While an ovarian teratoma is the most common neoplasm associated with anti-NMDA receptor encephalitis, physicians have implicated other neoplasms in a minority of patients [5]. Also, this syndrome can appear as an autoimmune disorder without any underlying tumor [6].

Although typically diagnosed in women, approximately $20 \%$ of cases have been males and most cases are in late adolescents and young adults [7]. The antibodies are usually detected in CSF/serum of young women with ovarian teratoma, who typically developed schizophrenia-like psychiatric symptoms, usually preceded by viral infection-like illness. Due to the challenges with identification, imaging, and diverse symptom presentation, this syndrome is often misdiagnosed. Even after the appropriate diagnosis is made there is often many important decisions and a waxing and waning presentation that requires ongoing monitoring.

\section{Case Discussions}

\section{Case 1}

Ms. A is a 25-year-old woman who was healthy, completely independent and was working on a full-time basis as an office cleaner. She had no prior significant medical history or any acute medical conditions. She had no psychiatric history but did have ADHD and a reading disorder and received special education throughout her 12 years of education. Her initial symptoms involved becoming more easily upset, agitated at times, and easily 
confused. She then started to become psychotic with hallucinations and suffered a seizure. She was acutely hospitalized and required antipsychotic medication, Lorazepam for anxiety, and divalproex sodium to manage her seizures. Other medical treatments included plasma exchange (PLEX), steroids, and Rituximab. She spent several weeks in the hospital and was found to have a uterine teratoma which was resected. Eight months later, at the time of her admission to a brain injury rehabilitation program, Ms. A remained symptomatic. At time of admission she exhibited impaired attention and short-term memory. Her Montreal Cognitive Assessment (MoCA) total score is $14 / 30$ and she is only oriented to self and year and recalled no words after a brief delay (0/5). Ms. A also exhibited affective lability and exhibited a very low frustration tolerance for the first 1.5 months of treatment. Through cognitive rehabilitation exercises and counseling along with a stable medication regime, she showed an improved frustration tolerance and improved and grossly functional cognitive skills.

\section{Case 2}

Ms. B: Ms. B is 23 years old and was healthy, fully independent, and working on a full-time basis in a factory when she first became symptomatic. She had no significant medical history but was 4 months pregnant when her symptoms began. She initially felt tired had become moodier and then started having seizures during her 2nd trimester. Approximately 6 months before this evaluation was her first seizure. When hospitalized with the seizure she was found to have a UTI. An MRI showed supratentorial white matter lesions thought related to NMDA encephalitis. She had multiple seizures during the hospitalization and had positive EEG findings. Specific antibody serum findings were not listed in the records. High dose Levetiracetam controlled the seizures, but Ms. B continued to exhibit impaired attention skills, short-term memory impairments, anxiety, and a labile mood. She was discharged home after three weeks and was then was admitted to outpatient rehabilitation. Initial evaluations found an unstable gait (berg balance test 40/56), generalized weakness, quick fatiguability, and mild cognitive impairment (MoCA score of 25/30). After 1 month of treatment she delivered a healthy full-term newborn and suffered multiple seizures during the birth process. The recovery that was made during month of treatment was lost and Ms. B had to essentially start her recovery and rehabilitation over. At this point in her treatment, Ms. A required three anticonvulsants: Clobazam, Lacosimide, Levetiracetam. These medications controlled her seizures and she gradually exhibited improved cognitive and physical functioning.

\section{Treatment Patterns}

Both of these patients had a pattern similar to most cases of this disorder. They went from being physically and emotionally healthy to needing acute inpatient care after the prodrome evolved into seizures and/or psychosis. During the care both patients received steroid treatment to help modulate their immune system. These included plasma exchange (PLEX), steroids, and Rituximab.

Both individuals had seizure's which were significant, but which were eventually controlled with anticonvulsants. Both individuals were prescribed psychotropic medications during acute care and needed to be maintained on these medications for several months. Both individuals needed outpatient brain injury rehabilitation services involving all disciplines. Each of these individuals returned to close to their baseline functioning at the conclusion of outpatient rehabilitation and they were still likely early enough post injury to continue to achieve further recovery over time. The medication issue in cases where someone is pregnant bring up the teratogenic issue and this.

\section{Course and Pathophysiology of Anti-NMDA}

Consistent with autoimmune dysfunction, patients suffering from paraneoplastic encephalitis associated with ovarian teratoma display antibodies for anti-N-methyl-D-aspartate (NMDA) receptors in CSF or plasma. The antibodies are for a subset of NMDA receptors that are preferentially expressed in the adult hippocampus and forebrain, which are brain regions involved in the pathogenesis of various psychiatric symptoms including psychosis. Moreover, the glutamatergic NMDA receptors are the major mediator of excitotoxicity and their dysfunction had been associated with neurologic disorders, but also with schizophrenia. Excessive release of glutamate in the prefrontal cortex and glutamate and dopamine dysregulation have been speculated to contribute to the development of schizophrenia-like psychosis and bizarre dyskinesias [8].

\section{Symptom Presentation}

Anti-NMDA encephalitis usually begins with a prodromal phase, followed first by prominent psychiatric symptoms or, less frequently, short-term memory loss, seizure, catatonia-like symptoms, dyskinesias and, secondly, by autonomic instability and central hypoventilation requiring intensive care. Distinct phases of illness have become increasingly appreciated and include a range of psychotic symptoms early in the course of the disease followed by more severe fluctuations in consciousness with neurologic [9].

Most cases develop seizures, followed by an altered mental status, and autonomic symptoms. The classic example of anti-NMDA receptor encephalitis consists of amnesia with mood change and depersonalization, involuntary movements, and seizures in a young woman eventually found to be harboring an ovarian teratoma. Then psychosis, seizures, involuntary movements, and autonomic instability often occur and eclipse the prodrome. Initial treating clinicians may suspect the onset of a major psychiatric disorder, herpes simplex encephalitis, phencyclidine (PCP) or another illicit drug intoxication.

\section{Diagnostic Testing}

Brain MRI is often unremarkable. CSF often reveals nonspecific changes, and EEG often shows diffuse delta slowing. In most cases the MRI initially shows no abnormalities; however, the CSF shows a lymphocytic pleocytosis. The CSF and serum contain antibodies to NMDA receptors. The antibody concentration, greater in the serum than CSF, roughly correlates with the severity of the illness. The CSF also contains oligoclonal bands, as it does in the CSF of cases of multiple sclerosis and other CNS inflammatory illnesses. 
This manuscript describes two individuals with NMDA encephalitis, Ms. A who developed it while 8 weeks pregnant and Ms. B who was found to have an ovarian teratoma, though continued to by symptomatic long after the teratoma removal.

\section{Conclusion}

Anti NMDA encephalitis may begin with subtle prodromal symptoms, but eventually causes significantly debilitating symptoms that require hospitalization. These cases demonstrate the need to consider NMDA encephalitis as a differential diagnosis in cases of acute behavior change in this age group. Traditional diagnostic procedures such as brain imaging, labs and toxicology screens may only provide limited information about what's going on with someone with this diagnosis. Both of these cases also show that this disorder carries a risk for recurrent seizures. Each individual showed positive effects from traditional neurorehabilitation strategies and counseling. Despite positive treatment effects these cases did not return to baseline functioning though may have subsequent to treatment. This is consistent with some research suggesting that symptoms can be prolonged and last from months to years [9].

NMDA encephalitis is a complex disorder with an unusual presentation. Both of these individuals reported that they were very much confused about their symptoms and really did not have any idea what to expect in terms of prognosis. Patient and family education were helpful in these cases and would likely be needed for most individuals with this disorder. Individuals with antiNMDA encephalitis often need potentially teratogenic medication (many anticonvulsants and psychotropic medications). So, there is a significant weighing of risks needed. Moreover, the fact that some psychotropic medications increase the risk of seizures, further complicates prescribing and effectively treating pregnant individuals with this disorder.

\section{Acknowledgemnet}

None.

\section{Conflict of Interest}

No conflict of interest.

\section{References}

1. Titulaer MJ, McCracken L, Gabilondo I, Glaser C, Iizuka T, et al. (2013) Treatment and prognostic factors for long-term outcome in patients with anti-NMDA receptor encephalitis: an observational cohort study. Lancet Neurol 12(2): 157-165.

2. Kumar MA, Jain A, Dechant VE, Saito T, Rafael T, et al. (2010) Anti-Nmethyl-D-aspartate receptor encephalitis during pregnancy. Arch Neurol 67(7): 884-887.

3. McCarthy A, Dineen J, McKenna P, Keogan M, Sheehan J, et al. (2012) Anti-NMDA receptor encephalitis with associated catatonia during pregnancy. J Neurol 259(12): 2632-2635.

4. Kokubun N, Komagamine T, Hirata K (2016) Pregnancy and delivery in anti-NMDA receptor encephalitis survivors. Neurol Clin Pract 6(5): e40-e43.

5. Hara M, Morita A, Kamei S (2012) Associated tumors in patients with anti-N-methyl-D-aspartate receptor (NMDAR) encephalitis. Rinsho Shinkeigaku 52(11): 979-981.

6. Kelley BP, Patel SC, Marin HL, Corrigen JJ, Mitsias PD, et al. (2017) Autoimmune Encephalitis: Pathophysiology and imaging: Review of an overlooked diagnosis. AJNR Am J Neuroradiol 38(6): 1070-1078.

7. Dalmau J, Gleichman AJ, Hughes EG, Rossi JE, Peng X, et al. (2008) AntiNMDA-receptor encephalitis: case series and analysis of the effects of antibodies. Lancet Neurol 7(12): 1091-1098.

8. Lau CG, Zukin RS (2007) NMDA receptor trafficking in synaptic plasticity and neuropsychiatric disorders. Nat Rev Neurosci 8(6): 413-426.

9. Kayser MS, Dalmau J (2011) Anti-NMDA Receptor Encephalitis in Psychiatry. Curr Psychiatry Rev 7(3): 189-193. 